\title{
Modelos mentales aplicados en la evaluación de aprendizajes por docentes de la Facultad de Filosofía de la Universidad de Cuenca
}

\author{
María Eugenia Maldonado \\ Centro de Posgrados, Facultad de Filosofía, Universidad de Cuenca, Ecuador. \\ Autor para correspondencia: maria.maldonado@ucuenca.edu.ec \\ Fecha de recepción: 30 de agosto de 2015 - Fecha de aceptación: 25 de octubre de 2015
}

\begin{abstract}
RESUMEN
La investigación pretendió demostrar la relación entre los modelos mentales de los profesores de carreras de formación docente y la evaluación de los aprendizajes a los estudiantes universitarios. El estudio se ubica en la Psicología Cognitiva, en el área del pensamiento docente, de manera particular en los modelos mentales, constituidos por creencias y concepciones que determinan las acciones de los profesores mayormente en base a su experiencia formativa, a sus subjetividades y menos en base a teorías científicas sobre evaluación. Los objetivos se dirigieron a establecer los enfoques pedagógicos sobre evaluación presentes en los modelos mentales de los profesores e identificarlos en relación con los componentes de la evaluación: objeto, función, instrumentos, momentos, agentes y destrezas mentales exigidas en la evaluación. El cumplimiento de los objetivos requirió de un tratamiento metodológico mixto. Para el primero se aplicó un diseño cuantitativo y para el segundo, uno cualitativo enfocado en las experiencias subjetivas de los docentes sobre sus prácticas de evaluación de los aprendizajes. Los resultados encontrados confirman la presencia de modelos mentales híbridos, es decir, formas de pensar respecto de la evaluación en base a mezclas y combinaciones entre elementos de origen y naturaleza teórica distinta, que afectan sustancialmente la práctica evaluatoria. Dichos híbridos mentales se constituyen de creencias y concepciones tradicionales y conductistas y menos desde una visión constructivista de la evaluación.
\end{abstract}

Palabras clave: Modelo mental, creencias, concepciones, evaluación de aprendizajes, profesores universitarios.

\begin{abstract}
This study demonstrates the relationship between mental models of teacher educators and learning assessment of college students. Theoretically, the study is based on Cognitive Psychology, more on teacher thinking, and the study in particular considers the mental models. These models are composed of beliefs and conceptions that determine the actions of professors mostly based on their training experience, their subjectivities and less based on scientific theories regarding the assessment. The objectives were to establish the pedagogical approaches about academic assessment existing on teachers' mental models on the one hand, and to identify them in relation to the assessment components: purpose, function, tools, time, agents and mental skills required, on the other hand. The methodology consisted of a mixed approach. For the first objective a quantitative design was used and a qualitative design for the second one, which was focused on the subjective experiences of professors about their practices of learning assessment. The findings confirm the presence of hybrids mental models. That is, ways of thinking about evaluation based on mixtures and combinations of elements of different theoretical origin and nature, which substantially affect the evaluation. Such mental hybrids are mostly traditional and behavioral and less focused on a critical view of the evaluation.
\end{abstract}

Keywords: Mental models, beliefs, conceptions, learning assessment, university professors. 


\section{INTRODUCCIÓN}

En el campo de la educación, se entiende a los modelos mentales como conjuntos de concepciones, creencias, saberes y sentidos comunes acerca del aprendizaje y la enseñanza, que influyen en el modo de comprender y actuar en el ámbito de desempeño de los docentes. Constituyen una red ideológica de teorías y creencias, la mayoría de las veces implícitas, que determinan el modo como el profesional docente da sentido a su mundo en general y a su práctica profesional en particular (Korthagen y Korthagen et al., citado por Pérez Gómez, 2012). Por la importancia que adquieren los estudios sobre el conocimiento implícito, en el ámbito de las investigaciones educativas actuales, se incluye el tratamiento de los modelos mentales, desde la óptica del "pensamiento del profesor" -teacher thinking- representado por un cúmulo de investigaciones provenientes de la pedagogía, la psicología, la etnografía (Ruffinelli et al., 2012). Esta línea investigativa considera que existe un conocimiento propio de los docentes, referido a saberes, convicciones, creencias, actitudes, reflexiones y expectativas, que se adquieren y utilizan en forma activa en la práctica profesional (Calderhead y Wanlin, citados por Ruffinelli et al., 2012).

Las concepciones del profesor "consisten en la estructura que cada docente da a sus conocimientos para posteriormente enseñarlos o transmitirlos a sus estudiantes" (García-Azcarate, citado por Tavarez \& Buduén, 2012). Se relacionan con las ideas y valoraciones que tienen los docentes sobre la naturaleza del conocimiento que enseñan (Ruffinelli et al., 2012) y con la manera como se aprende un conocimiento científico: naturaleza, estatus, relación con otros conocimientos, modo de producción, cambio, etc. (Porlán Ariza et al., 1998). Es decir, con una epistemología implícita o personal que surge a partir de las propias experiencias -generalmente formativas- y del contexto cultural en el que se desarrolla el individuo, incidiendo en las prácticas docentes y pedagógicas (Gorodokín, 2005; García et al., 2011). Las creencias, por su parte son preconceptos o premisas que las personas poseen acerca de lo que consideran verdadero. A diferencia del conocimiento proposicional, las creencias no requieren una condición de verdad contrastada (Marcelo \& Vaillant, 2011).

Pajares $^{1}$ (1992) uno de los investigadores más fecundos en el tema de las creencias, considera que estas se forman en edad temprana y tienden a perpetuarse, superando contradicciones causadas por la razón, el tiempo, la escuela o la experiencia; por tanto, cuanto más antigua sea una creencia, más difícil es cambiarla; tienden a mantenerse, pese a basarse en un conocimiento incompleto o incorrecto; conocimiento y creencias están interrelacionados, pero el carácter afectivo, evaluativo y episódico de las creencias se convierte en un filtro a través del cual todo nuevo fenómeno se interpreta; debido a su naturaleza y origen, algunas creencias son más indiscutibles que otras.

Tanto las concepciones como las creencias, componentes de los modelos mentales, se forman en el proceso de socialización de los estudiantes, en su paso por las instituciones educativas, situación que hace que interioricen saberes, reglas, pautas de acción que sus profesores llevaron a cabo con ellos. De allí, que los estudiantes de docencia cuando inician su formación profesional tienen ideas e imágenes más o menos consolidadas sobre lo que hacen los maestros, de tal manera que cuando ingresan en la educación superior tienen dificultad en cambiar las concepciones y creencias que configuran su modelo mental sobre la educación ${ }^{2}$ (López Fuentes, 2001; Feixas, 2010; Marcelo \& Vaillant, 2011; Pérez Gómez, 2012).

El criterio que justifica el estudio de los modelos mentales de los profesores, se fundamenta en el hecho de que es en la profesión docente, donde estos tienen mayor ocurrencia porque se establecen en base a creencias y concepciones adquiridas en los niveles inferiores de educación y durante el período de formación en educación superior (Rodríguez López, 1999; Blanco \& Barrantes, 2003; Gómez, 2008; Prieto, 2008; Tavarez \& Buduén, 2012). En consecuencia, la influencia de los modelos mentales en la práctica educativa es determinante pues funciona como un marco de referencia para los

\footnotetext{
${ }^{1}$ La mayor parte de los estudios e investigaciones sobre creencias, las caracterizan en referencia a los principios elaborados por Pajares.

${ }^{2}$ Ver conclusiones similares en la investigación La relación teoría-práctica en la formación docente para la innovación de la Educación General Básica. Universidad de Cuenca (2012).
} 
docentes, el que les permite comprender e interpretar sus experiencias y orientar sus acciones (Flores et al., 1997; Minakata, 2000; Perrenoud, 2001; Fortoul, 2008; Pérez Gómez, 2012).

La indagación de los modelos mentales de los profesores universitarios es un tema reciente en la investigación educativa. Se reportan trabajos de este tipo en los últimos veinte años, en los que figuran nombres como Pajares (1992), Porlán Ariza et al. (1998), Ponte (1999), Llinares (1991), Blanco \& Barrantes (2003), Gutiérrez (2005), Dodera et al. (2008). El énfasis de estos estudios está en establecer las relaciones entre el conocimiento del profesor, su construcción y transmisión en el contexto escolar. Complementariamente, otras investigaciones se centran en las ideas de los profesores acerca del conocimiento científico (naturaleza, estatus, relación con otros conocimientos, modo de producción, cambio, etc.) y las creencias pedagógicas que incluyen un amplio repertorio de aspectos relacionados con la enseñanza y el aprendizaje (Porlán Ariza et al., 1998). En la misma línea, las interpretaciones de lo que es un modelo mental varían en distintos estudios. Es así que son concebidas como preconcepciones y teorías "ingenuas" de los estudiantes en distintas áreas del conocimiento (Pozo, 1999; Blanco \& Barrantes, 2003; Amador Rodríguez et al., 2008) y también de los maestros (Alliaud, 2007; Fortoul, 2008). Así mismo, se concibe como "criterios de verdad" de los docentes para validar acciones educativas (Gutiérrez, 2005; Fortoul, 2008; Ontoria Peña et al., 2008), entre ellos, la evaluación de los aprendizajes.

Se encuentran estudios sobre la relación entre la enseñanza y las modalidades de la evaluación de los aprendizajes que los docentes eligen (Camilloni et al., 2007; Moreno Olivos, 2009; Ruffinelli et al., 2012). Las investigaciones reflejan que el resultado de la aplicación de prácticas inadecuadas e ideas erróneas sobre evaluación de aprendizajes, recae negativamente en los estudiantes (Gimeno Sacristán \& Pérez Gómez, 1998; Santos Guerra, 1999; Álvarez Méndez, 2001; Pérez Rivera, 2007; Prieto \& Contreras, 2008; Moreno Olivos, 2009).

Pese a la corta trayectoria de esta línea investigativa, la producción de estudios sobre modelos mentales del profesor universitario y aspectos puntuales sobre su práctica educativa, es abundante en lo que va de este siglo. Se reportan resultados de investigaciones sobre modelos mentales de los profesores y su incidencia en la evaluación de los aprendizajes a los estudiantes en universidades iberoamericanas (López Fuentes, 2001; Olmos Migueláñez, 2008; Pérez Rivera, 2007; Rueda Beltrán \& Torquemada González, 2008). Sin embargo, pese a este interés investigativo, no se encuentran estudios en este campo en el Ecuador. La relevancia de los estudios sobre modelos mentales se entiende desde la psicología cognitiva por el reconocimiento de las subjetividades del profesor como actor escolar. En este contexto, surge el interés por comprender el pensamiento del educador como un conocimiento propio que se adquiere y se utiliza de forma activa en su formación y práctica profesional (Bourdieu y Korthagen, citados por Pérez Gómez, 2012). Consecuentemente, las prácticas sobre la evaluación pueden funcionalizarse en base a los modelos mentales de los profesores, incidiendo en su concepción funcional, al concebirla como sinónimo de medición de la capacidad y del aprovechamiento de los alumnos centrado en la reproducción del conocimiento; entenderla como un requisito institucional o como ocurre frecuentemente, como un espacio de poder y control (Gimeno Sacristán \& Pérez Gómez, 1998; Santos Guerra, 1999; Álvarez Méndez, 2001; Pérez Rivera, 2007; Prieto, 2008; Moreno Olivos, 2009).

En razón de la importancia que en la práctica educativa tiene la evaluación de los aprendizajes, resulta significativo comprender cómo operan los modelos mentales de los profesores en la Facultad de Filosofía -formadores de docentes- con respecto a los criterios de evaluación de los aprendizajes para detectar congruencia con los enfoques: tradicional, conductista-positivista y constructivista, presentes en sus prácticas. Los objetivos específicos pretenden establecer los enfoques pedagógicos sobre evaluación presentes en los modelos mentales de los profesores e identificarlos en relación con los componentes de la evaluación: objeto, funciones, instrumentos, momentos y destrezas exigidas en la evaluación y explorar las formas de operar de los modelos mentales en torno a las prácticas evaluativas de los profesores. 


\section{MÉTODO}

La investigación tuvo un enfoque mixto; se utilizó un diseño exploratorio secuencial de tipo cuanticualitativo. Se recolectó información en amplitud sobre las prácticas de evaluación usadas por los profesores para encontrar congruencia con los paradigmas tradicional, conductista y críticoconstructivista en torno a los siguientes aspectos: objeto, función, instrumentos y momentos de la evaluación. Con tal propósito se aplicó un cuestionario estructurado ${ }^{3}$.

Las respuestas fueron procesadas en el paquete estadístico SPSS-Versión 18. Se planteó un análisis descriptivo general de las variables implicadas en el estudio, agrupando los resultados por bloques de contenidos: características del profesorado y planteamiento de la evaluación. Para el enfoque cualitativo se trabajó con un diseño de tipo fenomenológico enfocado en las experiencias individuales subjetivas de los docentes sobre sus prácticas evaluatorias y modelos mentales. Se utilizó la entrevista en profundidad para el análisis de discurso y sus significados, con lo que fue posible describir, comprender e interpretar la concepción y prácticas de la evaluación de los aprendizajes por parte de los docentes investigados.

El contenido de la entrevista consideró dos bloques temáticos: a) objetivo y función de la evaluación y, b) instrumentos, momentos, agentes de la evaluación y destrezas mentales exigidas en el proceso evaluatorio. Sobre todos ellos se pedía a los docentes su explicación.

\section{Población y muestra}

El total de docentes que trabajaban en la Facultad de Filosofía, Letras y Ciencias de la Educación (2013) fue de 118. De acuerdo con los objetivos del estudio y con el fin de identificar el universo y muestra definitiva, se excluyeron del estudio a 38 docentes. De este modo, se obtuvo como universo real 80 profesores, debido a que 3 se autoexcluyeron antes de la aplicación del cuestionario.

Muestra:

$$
\begin{array}{lll} 
& & \\
N=\frac{N \cdot Z^{2} \cdot p \cdot(1-p)}{e^{2} \cdot(N-1)+Z^{2} \cdot p \cdot(1-p)} \\
\mathrm{N} & = & 80 \text { profesores } \\
\mathrm{Z}^{2} & = & 95 \% \\
\mathrm{P} & = & 50 \% \\
\mathrm{E} & = & +-7 \% \text { (margen recomendado entre } 2-7 \%) \\
\mathrm{m} & = & 57 \text { profesores }
\end{array}
$$

Para las entrevistas en profundidad, los participantes se seleccionaron por aspectos definidos en la muestra criterial: formación profesional en docencia, que representen a cada una de las carreras de formación docente, así como las categorías de profesores (titulares y ocasionales), además de rangos de edad, género y años de docencia en la universidad. Como resultado, se obtuvo un grupo de siete docentes.

\section{RESULTADOS}

Se presenta dos tipos de análisis: cuantitativo de los datos de la encuesta y cualitativo mediante el análisis del contenido de las entrevistas.

\footnotetext{
${ }^{3}$ El cuestionario estructurado fue tomado, con autorización de Olmos Migueláñez (2008), en los aspectos pertinentes al estudio, de la encuesta "Opinión del profesorado sobre el uso de las tecnologías en evaluación de aprendizajes de alumnos universitarios" aplicada por Olmos Migueláñez en su tesis doctoral "Evaluación Formativa y sumativa de estudiantes universitarios".
} 
Tabla 1. Distribución de los docentes de acuerdo al género, edad, experiencia, categoría profesional y tipos de asignatura $(\mathrm{N}=57)$.

\begin{tabular}{llll}
\hline Variable & & $\mathrm{n}$ & $\%$ \\
\hline Género & Hombres & 30 & 52.6 \\
& Mujeres & 27 & 47.4 \\
\hline Edad (años) & Hasta 30 & 7 & 12.3 \\
& Entre 31 y 40 & 13 & 22.8 \\
& Entre 41 y 50 & 16 & 28.1 \\
& Entre 51 y 60 & 18 & 31.6 \\
& Más de 60 & 3 & 5.3 \\
\hline Años docencia & Menos de 1 año & 8 & 14.0 \\
& Entre 2 a 4 años & 17 & 29.8 \\
& Entre 5 a 10 años & 7 & 12.3 \\
& Entre 11 a 20 años & 10 & 17.5 \\
& Más de 20 años & 15 & 26.3 \\
\hline Categoría & Ocasional & 23 & 40.4 \\
profesional & Titular & 34 & 59.6 \\
\hline Tipo de asignatura & Básica & 11 & 19.3 \\
& Profesional & 33 & 57.9 \\
& Humanística & 7 & 12.3 \\
& Optativa & 1 & 1.8 \\
& No contesta & 5 & 8.8 \\
\hline
\end{tabular}

Fuente: Cuestionario aplicado a profesores.

Con respecto al género, porcentualmente se aprecia una distribución equitativa por esta condición, en tanto que por la edad el mayor porcentaje sitúa a los profesores entre 51 y 60 años, seguido de docentes entre 41 y 50 años. El porcentaje agregado de estas dos franjas de edad es $59.7 \%$, es decir, más de la mitad de los profesores tienen entre 41 y 60 años, lo que implica una importante trayectoria formativa y profesional acumulada. Los docentes que se ubican entre 31 y 40 años, representan un intervalo de edad también importante y son casi el doble de los que tienen hasta 30 años. Los resultados sobre años de docencia universitaria reflejan datos polarizados: $29.8 \%$ de profesores tienen entre 2 y 4 años de experiencia, en tanto que $26 \%$ de docentes acreditan más de 20 años en docencia universitaria. El promedio de años de trabajo en educación superior es de 11.4, tiempo que avala competencia en las destrezas básicas de enseñanza e implica que el profesor "posee destrezas de gestión de clase bien desarrolladas, destrezas en el uso de distintos modelos de enseñanza y de evaluación formativa de los estudiantes" (Leithwood, citado por Marcelo \& Vaillant, 2011). Pero asimismo, este período de actividad docente, permite un afianzamiento de opiniones, creencias y concepciones sobre enseñanza y evaluación de los profesores ${ }^{4}$.

El recuerdo de una profesora de la facultad sobre sus experiencias en docencia secundaria, refleja lo señalado:

La forma en que me evaluaron a mí era únicamente memorística, muchas de las cosas ni siquiera recuerdo ahora, ni sé para que me hicieron aprender con tantas horas de memorización. Ahora creo en la evaluación formativa y no punitiva, entonces prácticamente ahora no lo hago, pero sí puedo decir que lo hice y repliqué los mismos

\footnotetext{
${ }^{4}$ Pajares considera que "las creencias se forman en edad temprana y tienden a perpetuarse, superando contradicciones causadas por la razón, el tiempo, la escuela o la experiencia” (citado en Marcelo \& Vaillant, 2011).
} 
modelos con los me evaluaron a mí, los repliqué en los inicios de mi práctica docente (A14).

El mayor porcentaje -cerca del $60 \%$ - de profesores son titulares y en una proporción menor, pero también significativa $(40 \%)$ se encuentra la categoría ocasional. ${ }^{5}$ Al caracterizar este aspecto se encuentra que el profesorado está compuesto por sujetos con experiencia docente dada su condición, pero también con profesores que comienzan su práctica docente universitaria, situación que debe considerarse, pues de acuerdo a estudios (Marcelo, Manjón, García del Dujo y Vonk, citados por Marcelo \& Vaillant, 2011) un aspecto que caracteriza a las instituciones de docencia sobre todo universitaria, es su falta de preocupación acerca de la forma como los docentes se insertan en la enseñanza, pasando de su condición de estudiantes a profesores, cargados de modelos mentales formados con creencias que actúan como "filtros mediante los cuales dan sentido a los conocimientos y experiencias que van encontrando" (Feiman-Nemser, citados por Marcelo \& Vaillant, 2011). Resulta interesante advertir en las respuestas de los docentes entrevistados (ocasionales y titulares) percepciones sobre su escasa formación en el área de la evaluación, la débil reflexión sobre sus prácticas evaluativas y la creencia en la suficiencia de la experiencia lograda con los años para ser un experto evaluador.

Los siguientes fragmentos del discurso de los docentes así lo confirman:

Si tenemos varias deficiencias en cuanto a la formación en pedagogía... Entonces, yo creo que hemos ido aprendiendo en la práctica, un poco autoformándonos y también claro, aportando desde nuestras propias, no creo que podría llamar certezas, más bien intuiciones (E7).

Al tema de la evaluación no se le da tanta importancia y tanto peso (A7).

En realidad si un profesor no está bien formado copia modelos (de evaluación) que los compañeros comentan. (A7).

Con estas afirmaciones se confirma que el proceso de la evaluación en el mundo universitario está afectado por la creencia que da por sentado que cualquier persona, por el hecho de ser profesor, está capacitada para evaluar el aprendizaje de los alumnos (Moreno Olivos, 2009).

Respecto al tipo de asignaturas, el grupo de docentes investigado tiene a su cargo asignaturas básicas (19\%) y en mayor porcentaje, profesionales (57\%); es decir la actividad docente se cumple con elementos vertebradores de formación ${ }^{6}$, espacio propicio para la evidencia de los modelos mentales en general y de la evaluación en particular. Dado el carácter disciplinar de la formación universitaria, los profesores generalmente se ubican como conocedores expertos o especialistas de las asignaturas que tienen a su cargo. Como habitualmente ocurre, son "sus asignaturas". Consecuentemente sobre éstas, se desarrollan concepciones o marcos organizativos, con aspectos implícitos que provienen de los propios profesores, quienes aplican criterios e implementan prácticas docentes respecto de su enseñanza y evaluación, valorando de una u otra manera sus fines, sentido y trascendencia y determinando, en gran medida, la naturaleza de sus prácticas de enseñanza y evaluativas e incidiendo de manera crítica en los resultados de los procesos formativos (Prieto \& Contreras, 2008) ${ }^{7}$.

\footnotetext{
${ }^{5}$ Esta condición se debe básicamente a la elevada frecuencia de jubilaciones de profesores -a los 60 años de manera voluntaria y a los 70 de forma obligada- situación que requiere cubrir inmediatamente los puestos de trabajo con profesores ocasionales.

${ }^{6}$ Este resultado probablemente se debe a la estructura de las mallas curriculares, que desde el año 2009 se rigen por las disposiciones constantes en el Reglamento del Sistema de Créditos de la Universidad de Cuenca. Tipo de asignaturas: Humanísticas (6-12\%), Básicas (30-40\%), Profesionales (45-55\%), Optativas (6-10\%), Libre elección $(2-3 \%)$.

${ }^{7}$ La literatura de investigación consagrada al tema "aprender a enseñar" ha identificado algunas categorías de experiencias que influyen en las creencias y los conocimientos que los profesores desarrollan [...] entre ellas la experiencia escolar y de aula que incluye todas aquellas rutinas que se asimilan en condición de estudiante y contribuyen a formar una idea acerca de qué es enseñar y cuál es el trabajo del profesor (Marcelo \& Vaillant, 2011).
} 
El análisis subjetivo y retrospectivo de los profesores da cuenta de la importancia de la observación de sus modelos en la interiorización de ciertas prácticas cuando fueron estudiantes.

[...] al comienzo, en realidad uno reproduce las mismas prácticas de cómo fue evaluado cuando fue estudiante, eso lo haces cuando entras como profesor. Sí, al inicio de mi práctica docente, sí, pero conforme he ido capacitándome, formándome, las prácticas de evaluación son completamente diferentes (A14).

A partir del reconocimiento de la ausencia de conocimientos sobre evaluación, los profesores sugieren algunas soluciones que no están fundamentadas en la reflexión sobre sus prácticas, sino en procesos más bien externos, como la capacitación institucional.

Pero yo sí creo que sería bueno una capacitación masiva del profesorado en donde ellos entiendan la coherencia que debe existir entre el modelo educativo propiamente dicho y todos los elementos del modelo educativo, no solamente la parte de la metodología de enseñanza dentro de una clase, que sería el resultado final como el desempeño auténtico, sino también como saber si el estudiante ha llegado a ese desempeño auténtico y solamente de esa manera vamos a tenerlo con la evaluación formativa (A16).

Al respecto, Marcelo \& Vaillant (2011) consideran que la mayoría de programas de desarrollo profesional docente, establecen que la formación es el elemento capaz de producir cambios en las creencias y en los conocimientos de los profesores, lo que a su vez debería provocar una revisión crítica de las prácticas docentes en el aula y como consecuencia, producirse mejoras en los resultados de aprendizaje de los estudiantes. No obstante, Guskey y Spark, citados por en Marcelo \& Vaillant (2011) demostraron que los procesos de cambio no funcionan de esta manera y que los profesores cambian sus creencias no como consecuencia de participar en actividades de capacitación, sino a partir del contraste con las nuevas prácticas que se proponen desarrollar ${ }^{8}$.

El testimonio de una docente que ha participado de cursos de formación en la universidad, refleja la tendencia señalada.

Pienso que en toda transformación hay un periodo de incredulidad, creemos que no va a dar resultado las formas nuevas e innovadoras que ciertos profesores traen. En realidad, aquí va a tener que pasar un buen tiempo hasta que poco a poco se vaya regando un poco más las experiencias de algunos profesores que estamos trabajando de esa manera, o que se trabajen cursos de capacitación y poco a poco los profesores vayan aceptando estas nuevas formas de evaluación (A16).

Un elemento importante a señalarse es que en el discurso de los profesores sobre el papel institucional relacionado con el tema de la evaluación, consideran que el trabajo de las Juntas Académicas no afianza el sentido de ésta; la percepción de algunos docentes reconoce que en este importante espacio de socialización académica no se trata el tema de la evaluación de los aprendizajes.

Más o menos creo que todos llevamos la misma forma de evaluar, pero nunca hemos discutido en la Junta Académica ¿cómo evaluamos? y eso sería interesante. Nunca hemos discutido. Cada quien con su forma de ser y no estamos preguntando como evalúan (C8).

Las reuniones de juntas académicas no han servido para compartir ni para evaluar los instrumentos que están aplicando (D8).

Consecuentemente, para todos los profesores entrevistados, los instrumentos de evaluación son construcciones personales.

\footnotetext{
${ }^{8}$ En estudios locales Chacón et al. (2007) y Bijl van der (2008) señalan resultados similares de escasa innovación tras los procesos de capacitación docente.
} 
Tabla 2. ¿Para qué evaluar?

\begin{tabular}{lcccccccc}
\hline \multirow{2}{*}{ Criterios } & \multicolumn{2}{c}{$\begin{array}{c}\text { Más } \\
\text { importante }\end{array}$} & \multicolumn{2}{c}{ Importante } & \multicolumn{2}{c}{$\begin{array}{c}\text { Menos } \\
\text { importante }\end{array}$} & \multicolumn{2}{c}{ Total } \\
\cline { 2 - 12 } & $\mathrm{N}$ & $\%$ & $\mathrm{~N}$ & $\%$ & $\mathrm{~N}$ & $\%$ & $\mathrm{~N}$ & $\%$ \\
\hline $\begin{array}{l}\text { Cumplir con la obligación de } \\
\text { emitir una calificación }\end{array}$ & 12 & 7.02 & - & - & 14 & 8.19 & 26 & 15.20 \\
\hline $\begin{array}{l}\text { Detectar avances de los } \\
\text { estudiantes respecto a su } \\
\text { punto de partida }\end{array}$ & 20 & 11.70 & 11 & 6.43 & 9 & 5.26 & 40 & 23.39 \\
$\begin{array}{l}\text { Mejorar instrumentos } \\
\text { didácticos }\end{array}$ & 8 & 4.68 & 18 & 10.53 & 10 & 5.85 & 36 & 21.05 \\
\hline $\begin{array}{l}\text { Reflexionar sobre métodos } \\
\text { Replantear programa }\end{array}$ & 14 & 8.19 & 15 & 8.77 & 8 & 4.68 & 37 & 21.64 \\
\hline No contesta & 1 & 0.58 & 4 & 2.34 & 4 & 2.34 & 9 & 5.26 \\
\hline Total & 57 & 33.33 & 57 & 33.33 & 57 & 33.33 & 171 & 100.00 \\
\hline
\end{tabular}

Fuente: Cuestionario aplicado a profesores.

Con respecto a la función de la evaluación, en el grupo de docentes investigados, se aprecia una tendencia multi modal en casi todas las opciones de respuesta; esto es caracterizar un elemento como más importante, importante y menos importante, lo que se podría entender como la configuración de un modelo mental híbrido del que participan la gran mayoría de los docentes investigados. Sin embargo, predomina la función sumativa, con énfasis en detectar los avances de los estudiantes respecto a su punto de partida, pero en función de los resultados de su acción docente ${ }^{9}$ (23.39\%). Es decir, hay una tendencia por un enfoque conductista de la evaluación.

Es reiterativa la creencia de que la evaluación sirve para constatar el proceso de asimilación en los estudiantes como resultado de la enseñanza, con lo que se corrobora la presencia del modelo mental ligado al paradigma proceso-producto, que establece relaciones entre el proceso -conducta del profesor- y el producto -resultados medibles que alcanzan los alumnos-.

Las siguientes expresiones de los profesores dan cuenta de lo señalado:

(La evaluación) Sirve para medir, bueno, no solo para medir sino para darme cuenta si es que los estudiantes han asimilado o no algo que se les ha enseñado (A1).

Evaluar es para mí una constatación de una serie de actividades, de procesos que se les da a los estudiantes durante un determinado tiempo que corresponde a una asignatura (C1).

La evaluación está considerada como un instrumento de medida y ese instrumento, sirve para el docente más que para el estudiante, para saber cómo estoy impartiendo mis clases $(G 1)$.

Se encuentran porcentajes que orientan a pensar en una visión constructivista de la evaluación: reflexionar sobre los métodos (21.64\%), mejorar instrumentos didácticos (21\%), replantear el programa (13.45\%). Pero, del análisis de las entrevistas, se desprende que ninguno de los docentes que participaron, caracteriza el sentido de la evaluación desde un enfoque constructivista; por lo tanto no se encuentran razones que apoyen la reflexión sobre los métodos que usan o sobre repensar en el diseño del programa de la asignatura. Más bien se hallan afirmaciones reiteradas sobre la importancia de los contenidos, reflejo de la presencia de un enfoque tradicional en el proceso de enseñanza aprendizaje y en la evaluación.

\footnotetext{
${ }^{9}$ Di Franco (2003) asume la expresión didáctica ingenua de Pruzzo (1997) para referirse a la tendencia que concibe una relación lineal entre la enseñanza y el aprendizaje, en tanto que si la profesora dio el tema, el alumno lo aprendió.
} 
Las siguientes afirmaciones revelan este aspecto:

En la facultad hay un modelo tradicional [...] que está centrado en el profesor, en los conocimientos y no está centrado en lo que el estudiante puede hacer (A8).

Al profesor aquí en la Facultad de Filosofía, lo único que le interesa es que el estudiante conozca y sepa datos y conocimientos (A8).

Se encuentra que el $15 \%$ de los profesores investigados consideran la evaluación como una función certificadora, consideración que es un traductor eficiente de las respuestas a "notas". El modelo mental tradicional en este espectro de respuestas queda reflejado y se evidencia aún con más fuerza en las entrevistas.

Evaluar [...] para acreditar, es la única forma como se muestra que el estudiante puede pasar a un curso superior, no hay otra forma más (C2).

Nosotros tal vez por la reglamentación que tenemos por la institución, tenemos que asignar una calificación para decir si el estudiante sabe o no sabe, es lo único que nos obligan a nosotros a hacer (E2).

La función educativa, formativa y de mejora constante del proceso de enseñanza-aprendizaje se observa con menor fuerza.

Tabla 3. Momentos en los que se evalúa.

\begin{tabular}{lcc}
\hline Identificación de momentos & $\mathrm{N}$ & $\%$ \\
\hline Cuando introduce un nuevo tema o concepto & 1 & 1.8 \\
Momentos determinados reglamentariamente & 7 & 12.3 \\
A lo largo del proceso de aprendizaje & 45 & 78.9 \\
Tienen un cronograma particular de evaluación & 2 & 3.5 \\
Otros & 1 & 1.8 \\
No contesta & 1 & 1.8 \\
Total & 57 & 100 \\
\hline
\end{tabular}

Fuente: Cuestionario aplicado a profesores.

Los profesores consideran en un porcentaje alto (78.9\%) que evalúan y califican las producciones de los alumnos a lo largo de todo el proceso de aprendizaje, práctica que se corresponde con un modelo mental constructivista. Este dato revela que los docentes consideran más propicia la evaluación formativa que la sumativa y se evidencia en todos los niveles de edad de los profesores, en los rangos de años de docencia, en las categorías profesionales a las que pertenecen los docentes investigados y según los tipos de asignaturas que integran el currículo de las carreras en las que trabajan. Sin embargo, al revisar el contenido de las entrevistas, se advierte que la práctica de evaluación formativa no se encuentra en el modelo mental de la mayoría de profesores investigados.

Las siguientes expresiones así lo demuestran:

Tenemos que evaluar dentro de una unidad a ver si es que los estudiantes han cumplido o no con lo que se ha propuesto en la unidad (A3).

(Se evalúa) sobre la base de los contenidos por unidades (C3).

(Se evalúa) de acuerdo a cómo han ido alcanzando la mayoría, los logros de aprendizaje.... (B3).

El posicionamiento tradicional y conductista de la evaluación se reafirma además, al encontrar $12.3 \%$ de respuestas por el criterio de que es importante evaluar en los momentos determinados reglamentariamente.

La siguiente expresión de un docente da cuenta de este criterio.

En la facultad predomina el sistema del reglamento, evaluación, pruebas, lecciones, disertaciones, investigaciones, trabajo en clase, trabajos prácticos (C8).

Se encuentra un porcentaje bajo de respuestas en la opción evaluar cuando introduce un nuevo tema o concepto $(1.8 \%)$ que obedece a una visión constructivista y teóricamente refleja la importancia 
que se asigna a la evaluación diagnóstica, que parece no considerarse como importante entre el grupo de docentes investigados; detectar conocimientos previos de los estudiantes, no está en el modelo mental de los profesores como un elemento de evaluación constructivista, probablemente porque técnicamente, luego de su aplicación no se requiere asignar calificaciones o porque a partir del conocimiento previo de los estudiantes no es considerado como necesario. Se desvanece, la visión constructiva del conocimiento y la significatividad de los conocimientos previos.

Tabla 4. Agentes de evaluación.

\begin{tabular}{cccc}
\hline Agente de evaluación & & N & $\%$ \\
\hline Autoevaluación & Hasta el 25\% & 25 & 43.9 \\
& Hasta el 50\% & 14 & 24.6 \\
& Hasta el 70\% & 1 & 1.8 \\
& Más del 75\% & - & - \\
& No contesta & 17 & 29.8 \\
\hline Heteroevaluación & Hasta el 25\% & 4 & 7.0 \\
& Hasta el 50\% & 17 & 29.8 \\
& Hasta el 70\% & 20 & 35.1 \\
& Más del 75\% & 16 & 28.1 \\
& No contesta & - & - \\
\hline Coevaluación & Hasta el 25\% & 28 & 49.1 \\
& Hasta el 50\% & 8 & 14.0 \\
& Hasta el 70\% & - & - \\
& Más del 75\% & - & - \\
& No contesta & 21 & 36.8 \\
\hline
\end{tabular}

Fuente: Cuestionario aplicado a profesores.

Se constata que en el modelo mental de la totalidad de los profesores encuestados, predomina la creencia de considerar como fundamental la heteroevaluación. No hay una evaluación participativa generalizada y la figura del profesor como único agente evaluador es alta. Esta tendencia se ubica en el modelo mental tradicional de evaluar los aprendizajes.

No utilizamos coevaluación, la figura del profesor como evaluador es muy alta (B5).

Si bien frente a la posibilidad de realizar evaluación participativa se encuentran porcentajes importantes (43.9 y 49\%), no se halla una relación con instrumentos que evidencien la participación de los estudiantes a través de estrategias evaluativas de carácter alternativo como la autoevaluación, la evaluación entre iguales o la coevaluación. Consecuentemente no hay una práctica generalizada de coevaluación y autoevaluación, lo que convierte a la evaluación educativa en una actividad individual de cada profesor ${ }^{10}$. Algunas razones de esta creencia:

Hacemos, sí esa retroalimentación pero no llegamos a esa conciencia de autoevaluación que puedan tener y claro sigue estando en nuestras manos como docentes la evaluación total (B5).

Hay muchos (estudiantes) que tienen una autoestima altísima y se autoevalúan más allá de lo que deberían (E5).

\footnotetext{
${ }^{10}$ Similares resultados reportan Rodríguez Gómez (2010) y otros, pues concluyen que el tipo de evaluación que predomina es el de "evaluación tradicional" ya que sigue siendo un proceso diseñado, ejecutado y controlado esencialmente por parte del docente, no evidenciándose la participación de los estudiantes.
} 
Tabla 5. Instrumentos de evaluación.

\begin{tabular}{lcccccc}
\hline \multirow{2}{*}{ Instrumentos } & Nunca & $\begin{array}{c}\text { Casi } \\
\text { nunca }\end{array}$ & $\begin{array}{c}\text { Con } \\
\text { frecuencia }\end{array}$ & Siempre & $\begin{array}{c}\text { No } \\
\text { contesta }\end{array}$ & Total \\
\cline { 2 - 7 } & 19.3 & 14.0 & 35.1 & 12.3 & 19.3 & 100 \\
\hline $\begin{array}{l}\text { Lecciones orales } \\
\text { programadas }\end{array}$ & 47.4 & 22.8 & 7.0 & 0 & 22.8 & 100 \\
\hline $\begin{array}{l}\text { Lecciones orales } \\
\text { sorpresivas }\end{array}$ & 3.5 & 12.3 & 47.4 & 21.1 & 15.8 & 100 \\
\hline $\begin{array}{l}\text { Exposición oral de } \\
\text { temas }\end{array}$ & 10.5 & 21.1 & 31.6 & 21.1 & 15.8 & 100 \\
\hline $\begin{array}{l}\text { Lecciones escritas } \\
\text { programadas }\end{array}$ & 54.4 & 10.5 & 3.5 & 3.5 & 28.1 & 100 \\
\hline $\begin{array}{l}\text { Lecciones escritas } \\
\text { sorpresivas }\end{array}$ & 8.8 & 22.8 & 35.1 & 17.5 & 15.8 & 100 \\
\hline Pruebas objetivas & 8.8 & 17.5 & 50.9 & 8.8 & 14 & 100 \\
\hline Pruebas tipo ensayo & 14 & 22.8 & 31.6 & 15.8 & 15.8 & 100 \\
\hline Control de lectura & 12.3 & 12.3 & 38.6 & 24.6 & 12.3 & 100 \\
\hline $\begin{array}{l}\text { Resolución de } \\
\text { problemas }\end{array}$ & 7 & 21.1 & 35.1 & 15.8 & 21.1 & 100 \\
\hline Informes & 8.8 & 15.8 & 36.8 & 15.8 & 22.8 & 100 \\
\hline Organizadores gráficos & 15.8 & 24.6 & 22.8 & 15.8 & 21.1 & 100 \\
\hline Portafolio & 19.3 & 22.8 & 14 & 17.5 & 26.3 & 100 \\
\hline Simulaciones & 12.3 & 12.3 & 35.1 & 22.8 & 17.5 & 100 \\
\hline Prácticas & 21.1 & 10.5 & 22.8 & 28.1 & 17.5 & 100 \\
\hline Asistencia a clases & & & & & \\
\hline Funyyyyyy & & & & & & \\
\hline
\end{tabular}

Fuente: Cuestionario aplicado a profesores.

En general se aprecia el uso de variados instrumentos de evaluación. Los de mayor porcentaje son las pruebas objetivas y tipo ensayo. Son de alta aplicación las formas orales de evaluación: lecciones y exposiciones. La exposición oral de temas presenta un porciento acumulado de $68.1 \%$. Este instrumento en la práctica docente permite evaluar la comprensión de contenidos y se convierte en una forma de responsabilizar a los estudiantes por el desarrollo de temas ${ }^{11}$.

Pruebas, lecciones y exposiciones se inscriben en un modelo de evaluar centrado en la acción certificadora de aprendizajes. Las siguientes afirmaciones de los docentes así lo demuestran:

Siempre estoy pensando y ahora los exámenes, ¿qué voy a preguntar? (B6).

Al inicio del año lectivo les entrego, junto con el módulo de la asignatura, el cuestionario y guía de lectura, con todas las preguntas sobre las que versarán las pruebas de evaluación, orales y escritas. No hay sorpresas. Los alumnos saben a qué atenerse (F6).

Tenemos desglosado por unidad, inclusive los estudiantes saben cuáles son los objetivos de aprendizaje, cuales son los objetivos generales, cuales son los resultados del aprendizaje. Entonces sobre la base de eso se toma la prueba. [...] tomo preguntas y respuestas sobre contenidos de la asignatura, que sería como se evalúa (C6).

${ }^{11}$ De manera informal, los estudiantes señalan que no siempre son temas bien trabajados por los compañeros y que comúnmente quedan vacíos explicativos luego de las exposiciones; a esto se suma la tendencia generalizada de exponer mediante presentaciones en programas computarizados, que limitan la capacidad expositiva o argumentativa de los estudiantes. 
El control de lectura constituye también un instrumento usado con frecuencia importante (46.8\%) como una forma de vigilancia de lectura de contenidos. Formalmente, este procedimiento es similar a una prueba escrita. La resolución de problemas, es otro instrumento que considera que la práctica garantiza el aprendizaje. Este tipo de evaluación es usado habitualmente por los profesores en un porcentaje agregado de $63.2 \%$. Sin embargo hay alguien que piensa que, los ejercicios en clase son valorados como forma de control de asistencia (D3). La elaboración de informes como elemento de evaluación está presente en la mitad de los docentes; o obstante en la entrevista en profundidad, no se explicita su aplicación. El uso de organizadores gráficos es una práctica evaluatoria constructivista y procede de la teoría del aprendizaje significativo. El porcentaje acumulado de uso frecuente es de $62.6 \%$. En la conversación con los docentes, no se evidencia su aplicación ni la importancia en la evaluación. Instrumentos como el portafolio de evidencias lo aplican de forma habitual para la evaluación 38.6\%. Esta herramienta responde a un modelo constructivista de evaluación con aplicación baja entre los docentes. Las prácticas como elemento de evaluación, son tenidas en cuenta por el 57.9\% de docentes. Pese a su importancia, no es una modalidad generalizada de evaluación. Las simulaciones como componente de evaluación presentan un porcentaje acumulado de aplicación baja entre los profesores investigados, a pesar de constituirse en una forma de poner en juego en un ambiente reducido, las experiencias de una práctica docente posterior. La asistencia a clases, pese a tener un sistema especial de control, regulado por el Sistema de Créditos y el Estatuto Universitario, es usada como elemento acostumbrado de evaluación por la mitad de los profesores. Se advierte que los instrumentos de evaluación, básicamente, se dirigen a certificar los aprendizajes por parte de los docentes, pese a que algunas de estas herramientas, propician una evaluación constructiva.

Conviene indicar que, pese a que en el cuestionario se dio la opción de señalar otros instrumentos evaluatorios, no hay respuestas; por ejemplo, la observación y entrevista como elementos de evaluación cualitativa no se mencionan.

En la conciencia de algunos profesores está la importancia de usar instrumentos de evaluación con un sentido formativo; sin embargo, los obstáculos para el cambio son fuertes.

La evaluación formativa es lo que se debe hacer pero es una gran responsabilidad del profesor y consumen mucho el tiempo. El profesorado va a tener un poco de limitación o de resistencia a no querer utilizar estas formas de evaluación mucho más útiles, más amigables y mediadoras para el estudiante (A5).

Me gustaría solo aprobar o reprobar y llegar a la conclusión con el propio estudiante: apruebas o repruebas, y que ambos estemos de acuerdo, sería genial (B5).

El uso de los instrumentos señalados -los denominados tradicionales y los constructivistas- no refleja que la evaluación esté al servicio de los aprendizajes sino el aprendizaje al servicio de la evaluación (Pérez \& González, 2011).

Tabla 6. Destrezas mentales requeridas en la evaluación.

\begin{tabular}{lcccccc}
\hline Indicadores & Nunca & $\begin{array}{c}\text { Casi } \\
\text { nunca }\end{array}$ & $\begin{array}{c}\text { Con } \\
\text { frecuencia }\end{array}$ & Siempre & $\begin{array}{c}\text { No } \\
\text { contesta }\end{array}$ & Total \\
\hline $\begin{array}{l}\text { Elaboración de síntesis } \\
\text { personales }\end{array}$ & $8.8 \%$ & $10.5 \%$ & $47.4 \%$ & $12.3 \%$ & $21.1 \%$ & $100 \%$ \\
\hline $\begin{array}{l}\text { Aplicación de } \\
\text { conocimientos }\end{array}$ & - & $10.5 \%$ & $42.1 \%$ & $36.8 \%$ & $10.5 \%$ & $100 \%$ \\
\hline $\begin{array}{l}\text { Comprensión de } \\
\text { conceptos }\end{array}$ & $1.8 \%$ & $1.8 \%$ & $43.9 \%$ & $40.4 \%$ & $12.3 \%$ & $100 \%$ \\
\hline Análisis y reflexión & $35.1 \%$ & $28.1 \%$ & $17.5 \%$ & $1.8 \%$ & $17.5 \%$ & $100 \%$ \\
\hline Memorización & $5.3 \%$ & $19.3 \%$ & $35.1 \%$ & $22.8 \%$ & $17.5 \%$ & $100 \%$ \\
\hline Emisión de juicios & $3.5 \%$ & $15.8 \%$ & $33.3 \%$ & $26.3 \%$ & $21.1 \%$ & $100 \%$ \\
\hline $\begin{array}{l}\text { Identificación de lo } \\
\text { importante }\end{array}$ & & & & & $100 \%$ \\
\hline
\end{tabular}

Fuente: Cuestionario aplicado a profesores. 
El valor más alto producto de la acumulación de porcentajes se encuentra en la destreza comprensión de conceptos (84.3\%). Según la Taxonomía de Bloom (1956) y la revisión de Anderson \& Krathwohl (2001), la comprensión es un objetivo cognitivo bajo. Esta práctica se ubica en modelos mentales tradicional y conductista y apunta a la reproducción del conocimiento. Con similar porcentaje acumulado (84.3\%) se halla la destreza análisis y reflexión de contenidos, práctica constructivista que parece ser bastante exigida en los instrumentos de evaluación de los aprendizajes. La aplicación de conocimientos, con un porcentaje aglutinado de $78.9 \%$ representa la destreza de transferir los aprendizajes a nuevos contextos, capacidad altamente exigida en las evaluaciones a los estudiantes de la facultad. Pero los instrumentos de evaluación utilizados no reflejan este propósito.

En el grupo de docentes con incorporación reciente, se da un porcentaje de $7 \%$ que consideran los conocimientos como criterio de evaluación, lo que refleja una concepción tradicional de la evaluación. Con valores más bajos (47\%) se encuentra la destreza elaboración de síntesis personales y creativas, en el rango con frecuencia, pero disminuye notablemente en la opción siempre (12\%). El uso de esta destreza guarda relación con el predominio de las pruebas tipo ensayo. La descripción e identificación de aspectos importantes y valoración y emisión de juicios de valor tienen porcentajes acumulados bastante cercanos al $60 \%$, lo que determina que no sean capacidades exigidas de manera generalizada en la evaluación de los estudiantes.

La capacidad de memorizar y reproducir los contenidos, que se correspondería con el primer nivel del dominio cognitivo de Bloom, no es una exigencia contemplada mayormente en las evaluaciones, pero persiste un $29.3 \%$ de docentes que exigen esta capacidad, correspondiente con el modelo mental tradicional y conductista de evaluación.

La siguiente afirmación de una docente recoge este reconocimiento:

Sigue funcionando la memoria sí, sí que funciona así. Y cuando queremos que respondan a preguntas abiertas o argumentativas alli se cortan, no avanzan a hacer una argumentación (B5).

Los resultados del estudio demuestran que los docentes señalan toda una gama de destrezas intelectuales exigidas en sus evaluaciones, desde la comprensión de conceptos e ideas básicas de la disciplina hasta la emisión de juicios. Sin embargo, al relacionar este aspecto con los instrumentos y los momentos de evaluación, no se encuentra una relación adecuada, puesto que por el tipo de instrumentos que se usan en la evaluación, las destrezas más usadas son el conocimiento y la comprensión ${ }^{12}$.

\section{DISCUSIÓN}

Se concluye que el objetivo general de la investigación que apuntó a "comprender los modelos mentales de los profesores de la Facultad de Filosofía con respecto a los criterios de evaluación de los aprendizajes para detectar congruencia con los enfoques: tradicional, conductista-positivista y críticoconstructivista, presentes en sus prácticas" reporta una configuración híbrida, multi-modal en sus concepciones y creencias sobre evaluación. Esta configuración determina la presencia de modelos mentales "híbridos" es decir, formas de pensar respecto de la evaluación "como mezclas y combinaciones entre elementos de origen y naturaleza teórica distinta" (Sandoval Godoy, 2003) que afectan sustancialmente la práctica evaluatoria.

\footnotetext{
${ }^{12}$ Di Franco (2003) recurre a las expresiones de Santos Guerra para calificar esta práctica evaluatoria: "la forma de practicar la evaluación potencia o debilita un tipo de operaciones intelectuales". En términos de complejidad intelectual, las tareas podrían oscilar entre las menos potentes (memorizar) y las más potentes (crear).
} 
Dichos híbridos mentales ${ }^{13}$ se construyen con elementos tradicionales y conductistas en mayor grado y menos desde una visión constructivista de la evaluación, que entiende las producciones de los estudiantes como auténticos desafíos que permiten reconocer las dificultades, limitaciones y potencialidades del proceso del aprender.

La hibridación de los modelos mentales determina que el profesorado mantenga un conjunto de prácticas evaluatorias centradas en las siguientes concepciones y creencias:

a) Predominio tradicional de la evaluación en tanto sirve para constatar la asimilación de conocimientos como producto de la transmisión del docente; la "estructura de la enseñanza está determinada desde la evaluación" (Di Franco, 2003), es decir se enseña lo que se evalúa; el profesor pese a tener exigencias institucionales de planificar y evaluar por resultados de aprendizaje, insiste en evaluar los conocimientos que transmite; la evaluación es básicamente certificadora; el profesor es el principal agente evaluador; los exámenes son los instrumentos de recogida de información más usados y la destreza mental más exigida es la memorización.

b) Categorizar al estudiante como único responsable de sus calificaciones. Según Santos Guerra (1999) "los procesos atributivos que se plantean respecto al fracaso de los alumnos suelen focalizarse en comportamientos de éstos y no de los profesores o de la institución: son vagos, son torpes, están mal preparados, no saben estudiar, están desmotivados, no asisten a clases".

No sé si los estudiantes estudian para pasar, pero no necesariamente para conocer, para analizar, para estudiar... (D6).

Yo creo que (los estudiantes) no están conscientes de que cada evaluación y cada calificación responden también a un desarrollo de ellos, desarrollo en el dominio de la asignatura (E6).

Para los estudiantes la nota representa solo el pase de ciclo (B6).

La caracterización negativa de los estudiantes también es señalada por Di Franco (2003) pues acota en su investigación que "el $80 \%$ de los profesores consultados centra las responsabilidades del fracaso escolar en causas externas a las prácticas pedagógicas [...]. Fundamentalmente, son los alumnos (con su desidia, falta de interés, de participación) los responsables inevitables del fracaso. Generan así una mirada excluyente que no los involucra".

c) Constatar la débil importancia que los profesores asignan al proceso evaluativo, tanto en la formación inicial como en los años de servicio en la docencia universitaria. En el discurso de los docentes de la muestra, se recoge una sensación de insatisfacción sobre su formación docente inicial en este ámbito. Así mismo es recurrente el criterio de la experiencia acumulada como elemento que fundamenta la evaluación.

En mi época con una didáctica y una pedagogía no nos enseñaban a ser profesores.

Donde uno aprende a ser profesor es ahí parado frente al aula de clases (E9).

En algunos casos, inclusive se declara abiertamente un desconocimiento de modelos de evaluación, limitación que no se corrige desde una necesidad individual sino que se atribuye a la institución la responsabilidad de mejorarla.

Como modelo sistemático (sobre evaluación educativa) definitivamente, no conozco ninguno, para decir la verdad. Es un poco más bien las experiencias (C7).

${ }^{13}$ En el informe final de la investigación "La relación teoría-práctica en la formación docente para la innovación (Cuenca, 2012)" existe un hallazgo importante sobre modelos mentales híbridos. Al comparar los modelos mentales sobre aprendizaje y enseñanza en estudiantes que inician la carrera con los que termina, se encuentra que "la gran mayoría de estudiantes de ambos ciclos y de maestras (orientadoras de la práctica) tienen modelos mentales en los que se mezclan los dos paradigmas opuestos (modelos híbridos)". Las dos hibridaciones más comunes son, por una parte, la que combina un concepto de enseñanza centrada en el maestro (transmisión) con uno de aprendizaje centrado en el estudiante (construcción), y por otra, la que combina enseñanza como transmisión y mediación pedagógica al mismo tiempo. En ambos casos la constante es el concepto de enseñanza como transmisión. 
Yo creo que nos falta mucha capacitación en esa área, momentos de discusión, de compartir experiencias. Yo creo que falta mucho (D7).

En este sentido, hay coincidencia con los resultados que Rueda Beltrán \& Torquemada González (2008) señalan: "para los profesores universitarios no parece necesario definir el acto evaluativo con precisión, ni sus objetivos, el universo de referencia de las situaciones posibles a evaluar, los criterios, la forma de sintetizar la información disponible, la manera de interpretarla, o la utilización de los resultados". De ahí que la evaluación sea "un acto sincrético estrechamente ligado a la persona del evaluador y reconocido como tal, en la medida en que él es quien ha conducido el aprendizaje" (De Ketele, citado por Rueda Beltrán \& Torquemada González, 2008). Por lo anterior, "la evaluación está orientada primordialmente a certificar el éxito de los aprendizajes de los estudiantes" ${ }^{\prime 14}$, al priorizar una valoración estrictamente cuantitativa (Rueda Beltrán \& Torquemada González, 2008).

Semejante situación es confirmada también en los estudios de Pérez Rivera (2007); en las conclusiones de su investigación y en acuerdo con el pensamiento de Coll et al. (1993) señala que "es evidente que sin una práctica coherente y una formación o referente teórico conceptual claro y consciente por parte de los profesores sobre la evaluación, la función de ésta en el proceso de enseñanza-aprendizaje y las actividades para llevarla a cabo pierden mucho su razón de ser y pueden convertirse en prácticas evaluativas con una fuerte orientación tecnicista o que privilegian lo burocrático-administrativo sobre lo académico".

d) Frente a la falta de conocimientos claros sobre enfoques evaluativos, los profesores exigen mejoras a la universidad. Esta tendencia es señalada también por López Fuentes (2001) como la necesidad de los docentes de cursos para aprender a evaluar. Se ha señalado, que la capacitación tiene un escaso valor transformativo de concepciones y creencias y consecuentemente de cambio real en las prácticas de enseñanza, aprendizaje y evaluación si no va acompañado de la "mediación de los procesos de aplicación y reflexión en cuatro diferentes ámbitos: el dominio personal (conocimientos, creencias y actitudes del docente); el dominio de la práctica de enseñanza; el de las consecuencias en el aprendizaje de los estudiantes; y el dominio externo" (Clarke y Hollingsworth, citados en Marcelo \& Vaillant, 2011). Estos autores plantean que las mejoras en el desempeño profesional se producen tanto por la propia reflexión de los docentes como por la aplicación de nuevos procedimientos, es decir mediante procesos interconectados y no lineales.

\section{CONCLUSIONES}

Las principales conclusiones de este estudio tienen por objeto alertar sobre las limitaciones y consecuencias de una práctica evaluatoria de los aprendizajes fundamentadas en modelos mentales alejados o en contradicción con teorías pedagógicas que enfatizan en el carácter formativo de la evaluación educativa. Las concepciones y creencias que configuran los modelos mentales de los profesores, guían su accionar a partir de referentes interiorizados en el proceso de socialización del educador a lo largo de su trayectoria de formación. Este modelado implica la adopción de un conjunto de saberes, acciones y decisiones que actúan como filtros cuando el docente enfrenta la tarea de educar y evaluar haciendo evidente su propia concepción sobre el conocimiento, la educación, el aprendizaje, la evaluación. "Darla por sobreentendida, a partir de la experiencia vivida como alumno o acumulada como profesor, es ignorar la asunción de responsabilidades personales y descartar o esquivar los compromisos conceptuales, ideológicos y sociales a los que cada uno pueda llegar en y con su práctica docente" (Álvarez Méndez, 2001).

\footnotetext{
${ }^{14}$ Los resultados de la investigación de estos autores, indican que un alto porcentaje (48.5\%) asocian la evaluación a cuestiones de medición y retención del conocimiento, así como la aplicación de instrumentos de evaluación o exámenes hacia el alumno al finalizar su proceso de aprendizaje.
} 
Se debe advertir también que frente a las actuales exigencias sobre el rol del docente universitario, expuestas en un marco normativo que demanda competencias desde un enfoque conductista, es posible prever otras formas de hibridación de sus modelos mentales, a partir de la tensión entre el cumplimiento de la norma -referentes macro curriculares exigidos para la educación superior- la configuración mental sobre evaluación de los aprendizajes adquirida en su tránsito educativo y las tendencias constructivistas sobre evaluación que abogan por un proceso de orden formativo y procesual.

Ante ello, resulta indispensable la revisión de las concepciones y creencias que guían el accionar de los profesores universitarios para llegar al análisis de los referentes epistemológicos que hacen que enseñen y evalúen como lo hacen. De esta manera se comprenderá que la evaluación debe estar referida a como entienden el proceso de conocimiento, la relevancia y significatividad de lo que se enseña y cuáles son las mejores formas de informarse sobre los aprendizajes de los estudiantes.

Resulta necesario iniciar una búsqueda de alternativas para el abordaje de la evaluación, reconociendo la existencia de modelos teóricos que sugieren diversas funciones de la evaluación. Es primordial hacer un tratamiento sistemático sobre la evaluación de los aprendizajes reconociendo sus orientaciones y recuperando el sentido mismo de la evaluación formativa. Así, en la medida en que se desarrollen investigaciones que contrasten opiniones de docentes y alumnos con el análisis de instrumentos de evaluación, se tendrán mayores posibilidades de ofrecer alternativas de mejora hacia los programas de evaluación (Rueda Beltrán \& Torquemada González, 2008).

Finalmente, se considera que las conclusiones presentadas no pueden generalizarse dadas las características del abordaje cualitativo, cuyo objetivo es más bien comprensivo respecto de los modelos mentales de un grupo de docentes de una facultad y en una institución educativa. Los resultados llaman la atención sobre la necesidad de nuevas investigaciones sobre el pensamiento docente, la cultura escolar, las prácticas educativas desde la mirada de los estudiantes, para generar procesos formativos que trasciendan la capacitación instrumental y permitan apropiarse de una teoría de la evaluación educadora, en palabras de Di Franco (2003) "un tipo de evaluación que decodifica necesidades, andamia y sostiene la curiosidad y la comprensión posibilitando el aprendizaje". Esta evaluación se hace cargo de garantizar el derecho a aprender de los estudiantes.

\section{BIBLIOGRAFÍA}

Alliaud, A., 2007. La biografía escolar en el desempeño de los docentes. Buenos Aires: Escuela de Educación de la Universidad de San Andrés, pp. 5-28.

Alvarez Méndez, J.M., 2001. Evaluar para conocer, examinar para excluir. Madrid: Morata.

Amador Rodríguez, R., R. Gallego Badillo, R. Pérez Miranda, 2008. Desde que versiones epistemológicas contruyen modelos mentales los profesores en formación inicial: una investigación didáctica. Recuperado el 20 de septiembre de 2012 de http://www.pedagogica.edu.co/revistas/ojs/indez.php/TED/article.

Anderson, L.W., D.R. Krathwohl (Eds.), 2001. A taxonomy for learning, teaching, and assessing: A revision of Bloom's taxonomy of educational objectives. New York: Longman.

Bijl van der, B., 2008. Por qué no aprenden a innovar. Una auto investigación sobre la relación teoría-práctica en formación docente. Cuenca: Universidad de la Cuenca.

Blanco, L., M. Barrantes, 2003. Concepción de los estudiantes para maestro en España sobre la Geometría escolar y su enseñanza-aprendizaje. Revista del Comité Latinoamericano de Matemática Educativa, 6(2), 107-132.

Bloom, B., 1956. Taxonomy of educational objectives. Charlotte: Ed. David McKay Compay.

Camilloni, A., E. Cols, L. Basabe, S. Feeney, 2007. El saber didáctico. Buenos Aires: Paidós.

Chacón, H., F. Astudillo, J. Loaiza, 2007. La educación general básica en su laberinto. Anales, 51-69.

Coll, C., E. Martín, T. Mauri, 1993. El constructivismo en el aula.Disponible en http://www.terras.edu.ar. Buenos Aires: Graó. 
Di Franco, M.G., 2003. Representaciones docentes en la evaluación escolar. Recuperado el 11 de febrero de 2014 de http://www.biblioteca.unlpam.edu.ar/pubpdf/praxis/n14a16difranco.pdf.

Dodera, M., A. Burroni, M. Lázaro, 2008. Concepciones y creencias de profesores sobre enseñanza y aprendizaje de la matemática. Recuperado el 26 de octubre de 2012 de http://www.soarem.org.ar/Documentos/39/20.

Feixas, M., 2010. Enfoques y concepciones docentes en la Universidad. RELIEVE, 16(2), 1-27. Disponible en http://www.uv.es/RELIEVE/v16n2/RELIEVEv16n2_2.htm.

Flores, S.M., R.F. Macías, I.S. Santana, 1997. Las creencias de docentes mexicanos sobre el papel de la escuela y el maestro. Recuperado el 31 de marzo de 2012 de http://www.rieoei.org/de los lectores/106Macotela.Pdf, $24 \mathrm{pp}$.

Fortoul, B., 2008. La concepción de la enseñanza según los estudiantes del último año de la licenciatura en Educación primaria en México. Revista Perfiles Educativos, XXX(119), 72-89.

García, M.B., M. Sanz, S. Vilanova, 2011. Contenido y naturaleza de las concepciones de profesores universitarios de biología sobre el conocimiento científico. Recuperado el 5 de febrero de 2014 de http:www.reec.uvigo.es/volumen/volumen 10/ART2_vol10_N1.pdf.

Gimeno Sacristán, J., A. Pérez Gómez, 1988. Pensamiento y acción en el profesor: de los estudios sobre planificación al pensamiento práctico. Málaga: Universidad de Málaga.

Gómez, L.F., 2008. Las teorías implícitas de los profesores y sus acciones en el aula. Revista Electrónica Sinectica 30, 1-14.

Gorodokín, I., 2005. La formación docente y su relación con la espistemología. Recuperado el 25 de febrero de 2014 de http:// rieoei.org/deloslectores/1164Gorodokin.pdf.

Gutiérrez, R., 2005. Polisemia actual del concepto modelo mental. Consecuencias para la investigación didáctica. Revista de Investigações em Ensino de Ciências. Disponible en http://www.if.ufrgs.br/public/ienci.Consulta: 28 septiembre 2012, 209-226.

Llinares, S., 1991. La formación de profesores de matemáticas. Sevilla: GID.

López Fuentes, R., 2001. Creencias del profesorado universitario sobre Evaluación. Tesis doctoral. Disponible en: http://libro/ugr.es/handle/10481/4570. Consulta 4 marzo 2013. Granada: Universidad de Granada.

Marcelo, C., D. Vaillant, 2011. Desarrollo Profesional Docente ¿Cómo se aprende a enseñar? Madrid: Narcea,S.A.

Minakata, A., 2000. El maestro que aprende: educación para una nueva época. Revista Electrónica Sinéctica, 17, 14-23.

Moreno Olivos, T., 2009. Evaluación del aprendizaje en la universidad. Revista Mexicana de Investigación Educativa, 14(41), 563-591.

Olmos Migueláñez, S., 2008. Evaluación formativa y sumativa de estudiantes universitarios: Aplicación de las tecnologías a la evaluación educativa. Recuperado el 27 de Noviembre de 2012 de http://gredos.usal.es/jspui/handle/10366/18453. Repositorio Documental de la Universidad de Salamanca.

Ontoria Peña, A., J.P. Gómez, A. Molina Rubio, 2000. Los modelos mentales: referentes de la acción o conducta. Madrid: Narcea.

Pajares, M.F., 1992. Teachers' beliefs and educational research:Cleaning up a messy construct. Review of Educational Research, 62(3), 307-332.

Pérez Gómez, A., 2012. Educarse en la era digital. Madrid: Morata.

Pérez Rivera, G., 2007. La evaluación de los aprendizajes. Recuperado el 27 de enero de 2014, de http://www.redalyc.org/articulo.oa?id=34004803.

Pérez, L., D. González, 2011. Dime cómo evaluas y te diré qué enseñas. Un análisis teórico sobre las relaciones entre la evaluación del aprendizaje y la enseñanza-aprendizaje de la justicia social. Revista Iberoamericana de Evaluación Educativa, 135-148.

Perrenoud, P., 2001. La formación de los docentes en el Siglo XXI. Revista de Tecnología Educativa, 503-523. 
Ponte, J.P., 1992. Concepções dos professores de matemática e processos de formação. Disponible en http://www.educ.fc.ul.pt/docentes/jponte.

Porlán Ariza, R., A. Rivero García, R. Martín del Pozo, 1998. Conocimiento profesional y epistemología de los profesores, II: Estudios empíricos y conclusiones. Enseñanza de la Ciencias, 16(2), 271-288.

Pozo, J.I., 1999. Más allá del cambio conceptual: el aprendizaje de la ciencia como cambio representacional. Revista Electrónica de Enseñanza de las Ciencias, 5(3), 513-520.

Prieto, M., 2008. Creencias de los profesores sobre evaluación y efectos incidentales. Revista de Pedagogía, 29(84), 123-144.

Prieto, M., G. Contreras, 2008. Las concepciones que orientan las prácticas de evaluación de los profesores: un problema a develar. Recuperado el 5 de febrero de 2014 de http://www.redalyc.org/pdf/1735/173514136914.pdf.

Rodríguez López, J.M., 1999. Las teorías implícitas sobre la enseñanza de los profesores en formación antes de las prácticas: el caso de Alicia. Recuperado el 15 de diciembre de 2012 de http://rabida.uhu.es/dspace/habdle/10272/318.

Rueda Beltrán, M., A. Torquemada González, 2008. Las concepciones sobre "evaluación" de profesores y estudiantes: sus repercusiones en la evaluación del desempeño docente. Recuperado el 27 de enero de 2014, de http://www.redalyc.org/articulo.oa?id=34005309.

Ruffinelli, A., M.J. Valdebenito, M.T. Rojas, L. Sepúlveda, 2012. Procesos de enseñanza-aprendizaje desde la perspectiva de los profesores en Chile. Informe de la Universidad Alberto Hurtado/Facultad de Educación/Centro de Investigación y Desarrollo de la Educación CIDE, Chile: Santiago, 93 pp. Disponible en http://www.cide.cl/documentos/Procesos_de_ensenanza _aprendizaje_desde_la_perspectiva_delos_profesores.pdf.

Sandoval Godoy, S., 2003. Hibridación Social: un modelo conceptual para el análisis de la región y el territorio. Revista Región y Sociedad, XV(28), 48-79.

Santos Guerra, M.Á., 1999. 20 paradojas de la evaluación del alumnado en la Universidad española. Revista Electrónica Interuniversitaria de formación del Profesorado, 1(2), 370-391.

Tavarez, A., I. Buduén, 2012. Principales ideas y concepciones acerca de las creencias de los profesores en el proceso de enseñanzar-aprendizaje. Revista de Pedagogía Profesional, 10(2), 18. 